\title{
Hormonal modulation of larval begging and growth in the burying beetle Nicrophorus vespilloides
}

\author{
TARA C. CROOK*, THOMAS FLATT† \& PER T. SMISETH* \\ *Faculty of Life Sciences, The University of Manchester \\ $\dagger$ Department of Ecology and Evolutionary Biology, Brown University \\ (Received 19 January 2007; initial acceptance 14 March 2007; \\ final acceptance 2 April 2007; published online 25 September 2007; MS. number: 9246)
}

\begin{abstract}
Recent studies on birds show that two steroid hormones, testosterone and corticosterone, stimulate nestling begging and growth. Here, we seek to investigate whether juvenile hormone, a major regulatory insect hormone, has similar effects on larval begging and growth in insects. To this end, we experimentally elevated larval juvenile hormone levels by topical application of methoprene, a potent and stable synthetic juvenile hormone analogue, and monitored effects on larval begging and growth in the burying beetle Nicrophorus vespilloides. In this species, larvae feed partly by begging for predigested carrion from parents and partly by self-feeding. We showed that elevated juvenile hormone levels stimulate larval begging, suggesting that juvenile hormone in insects could have a similar function to that of testosterone and corticosterone in birds. We also showed that elevated juvenile hormone levels have a negative effect on larval growth and that this negative effect occurs regardless of whether larvae forage by begging or by self-feeding. This finding shows that the effects of juvenile hormone on larval growth are independent of the effects on begging, suggesting that the mechanisms by which juvenile hormone affects offspring growth in insects differ from those by which testosterone and corticosterone affect growth in birds.
\end{abstract}

(C) 2007 The Association for the Study of Animal Behaviour. Published by Elsevier Ltd. All rights reserved.

Keywords: begging; burying beetle; growth; insects; juvenile hormone; Nicrophorus vespilloides; signalling of need

In many animal species, offspring solicit resources from their parents by engaging in conspicuous begging displays (Kilner \& Johnstone 1997). Game theory suggests that conspicuous offspring begging signals provide an evolutionarily stable resolution of within-family conflicts of interest over resource allocation because such signals are costly and therefore provide parents with reliable information on offspring need (Godfray 1991, 1995). Empirical studies in diverse taxa suggest that begging levels reflect offspring hunger and that parents provide resources in relation to begging levels (Kilner \& Johnstone 1997). Recent studies on different birds show that hormones play an important role in stimulating nestling begging effort, thereby enhancing offspring growth (e.g. Schwabl

Correspondence: P. T. Smiseth, Faculty of Life Sciences, Michael Smith Building, The University of Manchester, Oxford Road, Manchester M13 9PT, U.K. (email: per.t.smiseth@manchester.ac.uk).T. Flatt is at the Department of Ecology and Evolutionary Biology, Division of Biology and Medicine, Brown University, Box G-W, 80 Waterman Street, Providence, RI 02912, U.S.A.
1996; Kitaysky et al. 2001; Eising \& Groothius 2003; Goodship \& Buchanan 2006). Here, we seek to investigate whether the endocrine system in insects has a similar effect on larval begging.

In birds, testosterone and corticosterone, two steroid hormones, stimulate nestling begging behaviour. For example, in canaries, Serinus canaria, and black-headed gulls, Larus ridibindus, levels of maternally derived testosterone in the egg yolk are positively correlated with begging and growth after hatching (Schwabl 1996; Eising \& Groothius 2003). In pied flycatchers, Ficedula hypoleuca, levels of circulating endogenous testosterone in the nestlings correlate positively with begging and fledging success (Goodship \& Buchanan 2006), and in black-legged kittiwakes, Rissa tridactyla, experimentally elevated levels of corticosterone stimulate begging (Kitaysky et al. 2001). By contrast, levels of maternally derived testosterone in the egg yolk of European starlings, Sturnus vulgaris, correlate positively with growth but negatively with begging at hatching (Pilz et al. 2004). Thus, while there is good evidence that hormones affect begging and growth in birds, 
the detailed causal relationship between circulating hormones, begging, and growth shows interspecific variation.

To date, studies on the hormonal modulation of offspring begging and growth have exclusively focused on birds. This is surprising given that offspring begging occurs in a wide range of animal taxa, including some insects (Rauter \& Moore 1999; Smiseth \& Moore 2002; Kaptein et al. 2005; Kölliker et al. 2006). Insects offer a particularly interesting model for studying hormonal modulation of begging and growth because insects possess a hormone system quite distinct from that of vertebrates (Nijhout 1994). Besides many specialized peptide neurohormones, insects have two major regulatory hormones (1) juvenile hormone, a lipid-like sesquiterpenoid, and (2) 20-hydroxy-ecdysone, a steroid, both of which are involved in the regulation of numerous aspects of physiology, development, life history, and behaviour (Nijhout 1994; Flatt et al. 2005).

Here we focus on the potential role of juvenile hormone in modulating larval begging and growth in the burying beetle Nicrophorus vespilloides. This species, like other members of the same genus, breeds on carcasses of small vertebrates (Eggert \& Müller 1997; Scott 1998). Larvae forage partly by begging for predigested carrion from the parents and partly by self-feeding from the carcass (Smiseth \& Moore 2002; Smiseth et al. 2003). Juvenile hormone is a versatile hormone that plays a regulatory function in numerous contexts in insect larvae, including the onset of metamorphosis (Williams 1956; Nijhout 1994), growth of imaginal tissues (Emlen \& Allen 2003), metabolic activity (Sláma \& Hodková 1975) and immune function (Rantala et al. 2003). In other insects, juvenile hormone titers reflect offspring nutritional state (e.g. Schal et al. 1997). This makes juvenile hormone a particularly attractive candidate hormone for regulating begging and growth because, in $N$. vespilloides, begging levels reflect nutritional state (Smiseth \& Moore 2004), and begging has a positive effect on growth (Lock et al. 2004). In adult burying beetles, juvenile hormone is positively correlated with parental care towards begging larvae (Panaitof et al. 2004; Scott \& Panaitof 2004; Trumbo \& Robinson 2004). However, whether juvenile hormone also plays a role in modulating larval begging and growth is unknown.

The aim of our study was to investigate whether juvenile hormone could play a similar role in modulating larval begging and growth in insects as testosterone and corticosterone in birds. A recent study shows that juvenile hormone can play a similar role in insects as testosterone and corticosterone in vertebrates at least with respect to effects on immune function (Rantala et al. 2003). However, juvenile hormone is structurally different from testosterone and corticosterone and appears to play a regulatory role in a multitude of contexts in insects, which in vertebrates are regulated by different hormones (Nijhout 1994). If juvenile hormone plays a similar role in insect larvae as testosterone and corticosterone in nestling birds, we expect that elevated juvenile hormone levels stimulate begging and enhance growth. To test this expectation, we experimentally elevated juvenile hormone levels of $N$. vespilloides larvae and monitored effects on larval begging and growth. We next investigated the causal relationship between juvenile hormone levels, begging, and growth. In birds, testosterone and corticosterone might enhance nestling growth and survival by stimulating begging (Schwabl 1996; Kitaysky et al. 2001) or by inhibiting ineffective and costly begging (Pilz et al. 2004). If juvenile hormone has a similar effect as testosterone and corticosterone in birds, we expect that increased hormone levels affect growth when larvae beg for resources from the parents but not when they forage by self-feeding. To test this expectation, we manipulated the presence or absence of parents and monitored the effects of juvenile hormone on growth.

\section{METHODS}

\section{General Procedures}

Adult beetles originated from an outbred laboratory population. For use in the experiments, we randomly selected pairs of nonsibling virgin male and female beetles. Once pairs had been selected, they were moved to a transparent container $(17 \times 12 \mathrm{~cm}$ and $6 \mathrm{~cm}$ high $)$ filled with a $2 \mathrm{~cm}$ layer of moist soil. We provided each pair with a previously frozen mouse carcass (Livefoods Direct Ltd, Sheffield, U.K.), with a mean size \pm SD of $24.5 \pm 2.1 \mathrm{~g}(\mathrm{~N}=65)$.

Approximately $60 \mathrm{~h}$ after a pair had been set up for breeding, the female was transferred to a new container along with the carcass. The male parent was removed at this stage because male care has no effect on offspring growth or survival under laboratory conditions (Smiseth et al. 2005). The eggs were left to hatch in the old containers, which were searched for any newly hatched larvae four times each day. We used newly hatched larvae to generate standardized broods of 20 same-aged larvae. This brood size is well within the natural variation for this species (mean $\pm \mathrm{SD}$ : $21 \pm 10$ larvae; range 2-47 larvae; Smiseth \& Moore 2002). We standardized brood size and age composition because begging is affected by variation in both brood size (Smiseth \& Moore 2002) and age (Smiseth et al. 2003).

\section{Juvenile Hormone Treatment}

To investigate whether juvenile hormone plays a role in modulating larval begging and growth in $N$. vespilloides, we experimentally elevated larval juvenile hormone levels by topical application of methoprene (Sigma-Aldrich Company Ltd, Poole, Dorset, U.K.) to the back of the larvae just below the head using a pipette. Methoprene is a potent and stable synthetic juvenile hormone analogue that is widely used in insect endocrinology because it reliably mimics juvenile hormone action (Wilson 2004). The manipulations were always conducted $24 \mathrm{~h}$ after hatching, that is, during the second larval instar, corresponding to the age of the peak in begging and food provisioning (Smiseth et al. 2003).

To determine which concentrations of methoprene to use in our experiments, we first conducted a pilot experiment in which we randomly divided larvae of 11 broods into four within-brood treatment groups: (1) $50 \mu \mathrm{g}$ of 
methoprene dissolved in $0.5 \mu \mathrm{l}$ of $95 \%$ ethanol, (2) $25 \mu \mathrm{g}$ of methoprene dissolved in $0.5 \mu \mathrm{l}$ of $95 \%$ ethanol, (3) $5 \mu \mathrm{g}$ of methoprene dissolved in $0.5 \mu \mathrm{l}$ of $95 \%$ ethanol, and (4) $0.5 \mu \mathrm{l}$ of $95 \%$ ethanol (i.e. a solvent control). In the pilot experiment, we monitored effects of juvenile hormone treatment on larval growth because it is easier to measure and less variable than larval begging. We used repeated measures GLM to test for effects of hormone treatment on larval growth (see 'Statistical Analyses' below for details). The pilot experiment showed that juvenile hormone treatment had a significant effect on larval growth (repeated measures GLM: within-subjects effects: $F_{6,60}=4.07, P=0.002$ ). Post hoc pairwise comparisons showed that there was a significant difference in growth between larvae assigned to the solvent control and larvae treated with $50 \mu \mathrm{g}$ methoprene $\left(F_{2,20}=7.31, P=0.004\right)$, while there were no significant differences in growth between larvae assigned to the solvent control and larvae treated with either $25 \mu \mathrm{g}$ or $5 \mu \mathrm{g}$ methoprene $\left(F_{2,20}=1.36\right.$, $P=0.28$ and $F_{2,20}=0.27, P=0.77$, respectively).

In the behavioural part of our experiment, which was conducted under red light, we would only be able to distinguish between three markings (Smiseth \& Moore 2004; also see below). Thus, we were limited to three within-brood treatments in our final experiments. Based on the pilot experiment, we decided to focus on the following three treatments. (1) Treatment with $50 \mu \mathrm{g}$ of methoprene dissolved in $0.5 \mu$ l of $95 \%$ ethanol (hereafter, termed 'high juvenile hormone treatment'); this treatment was included because the pilot experiment showed that it had a significant effect on larval growth (see above). (2) Treatment with $25 \mu \mathrm{g}$ of methoprene dissolved in $0.5 \mu \mathrm{l}$ of $95 \%$ ethanol (hereafter, termed 'low juvenile hormone treatment'); this treatment was included because it represents an intermediate concentration between the high juvenile hormone treatment and the solvent control. (3) Treatment with $0.5 \mu \mathrm{l}$ of $95 \%$ ethanol (hereafter, termed 'solvent control').

For hormonal manipulation, we removed 12-15 larvae, depending on the number of larvae in the brood still alive at the time of manipulation, and assigned them randomly to the high juvenile hormone, low juvenile hormone, or solvent control treatments such that each treatment group contained 4-5 larvae. In all broods, at least 16 of the 20 original larvae placed with the female had survived to the age at which they were treated. We also left 4-5 larvae on the carcass with the female to ensure that she did not desert the brood while the other larvae were subject to treatment. While removed from the carcass, larvae were kept in small plastic containers lined with moist paper to prevent desiccation.

\section{Larval Begging}

We set up 45 broods to investigate the effect of juvenile hormone on begging. Before being treated, larvae were marked with a small piece of coloured cotton attached with superglue to the back just below the head (Smiseth \& Moore 2004). Larvae assigned to different treatments were marked with different colours (i.e. black, dark red, or white). In broods where some larvae have been subject to marking and handling, there is no difference in time spent begging between larvae that have been handled and larvae that have not been handled (Smiseth \& Moore 2004). However, larvae in broods where some larvae have been handled tend to spend less time begging than larvae in where no larvae have been handled (P. T. Smiseth, unpublished data).

To ensure that larvae had absorbed the hormone, we kept larvae of each treatment group in separate containers for $6 \mathrm{~h}$. During this time, larvae were provided with food in the form of a small piece of carrion. After marking and treatment, we placed larvae in randomized order back on the carcass. We allowed a 15-min period to allow larvae to settle down before conducting the behavioural observations.

Observations were done under red light using instantaneous scan sampling (Martin \& Bateson 1986) every 1 min for a total period of $30 \mathrm{~min}$. At each scan, we counted the number of begging larvae in each treatment group. A larva was considered to beg when raising its head towards the parent while waving its legs or touching the parent (Rauter \& Moore 1999). Because begging only occurs when parents are near larvae (Rauter \& Moore 1999; Smiseth \& Moore 2002), we also recorded the number of scans in which the female was near the larvae; that is, less than the width of its pronotum away from the larvae (Rauter \& Moore 1999). From this, we calculated the average percentage time spent begging for each larva in a given treatment as bpi $=\left(\sum b / L\right)(100 / p)$, where $\sum b$ is the total number of begging events for a given treatment in an observation session, $L$ is the number of larvae in a given treatment group for a given brood, and $p$ is the number of scans during which the female was near the larvae (Smiseth et al. 2003; Smiseth \& Moore 2004).

\section{Larval Growth}

We set up 20 broods to investigate the effects of juvenile hormone on growth of begging larvae. To examine whether hormone treatment had an effect on growth when larvae foraged by self-feeding rather than begging, we set up an additional 20 broods where both parents were removed when the offspring were $24 \mathrm{~h}$ of age. Before being treated, larvae assigned to different treatments were marked by cutting the outer part of either the left hindleg, the right hindleg, or the right middle leg (Rauter \& Moore 2002). Immediately after marking, larvae were weighed to the nearest $0.0001 \mathrm{~g}$. After marking and treatment, we placed larvae back on the carcass in random order with respect to treatment. To monitor effects of the hormone treatment on growth, we weighed larvae to the nearest $0.0001 \mathrm{~g}$ at $24 \mathrm{~h}( \pm 15 \mathrm{~min})$ intervals until they were 96-h-old, the age at which the larvae reach maximum body mass before dispersing from the carcass (Smiseth et al. 2003).

\section{Statistical Analyses}

For the analysis on begging, we used data from 30 out of the 45 broods that were initially set up. We excluded 15 broods from this analysis because the female parent 
remained immobile underneath the carcass during the entire observation session. Broods where the female parent remains immobile underneath the carcass provide no data on larval begging because begging only occurs when parents are near larvae (Rauter \& Moore 1999; Smiseth \& Moore 2002). We used repeated measures GLM to test for effects of juvenile hormone treatment on begging and growth because our experiments were based on a split-brood design (von Ende 2001; Howell 2002). In these models, brood is the subject, and juvenile hormone treatment is a within-subjects factor with three levels (high juvenile hormone, low juvenile hormone and solvent control treatments). In the analysis on growth, we entered age as a within-subjects factor with four levels $(24,48,72$ and $96 \mathrm{~h})$. These models treat growth as the change in body mass with age. Because the sole interest in our study was to test for effects of hormone treatment on growth, we only report test statistics for the effect of juvenile hormone treatment on growth. In all analyses, we used average values across all larvae of a given treatment within each brood. We tested the assumption of sphericity using Mauchly's test (von Ende 2001; Howell 2002). Whenever the assumption of sphericity was violated, we used the Greenhouse-Geisser correction, which corrects for such violations by adjusting the degrees of freedom (von Ende 2001; Howell 2002). We used trend analysis to examine whether the effect of juvenile hormone treatment on larval begging and growth could be best explained by a polynomial (i.e. linear or quadratic) function. Trend analysis is particularly well suited for exploring further details in experimental designs with multiple ordinal within-subjects factors (Howell 2002), such as juvenile hormone treatment in our experiment. All variables used in the statistical analyses were either normally distributed or subject to arcsine square-root transformations to achieve normality and homoscedasticity. Data were analysed using SPSS 11 (SPSS Inc., Chicago, IL, U.S.A.) for Macintosh. All tests were two tailed.

\section{RESULTS}

\section{Larval Begging}

Juvenile hormone treatment had a significant effect on the time spent begging by the larvae (repeated measures GLM: within-subjects effects: $\left.F_{2,58}=3.31, P=0.044\right)$. As expected if juvenile hormone stimulated larval begging, experimental larvae treated with juvenile hormone spent more time begging than control larvae treated with solvent only (Fig. 1). Trend analysis revealed a significant linear component to the function describing the effect of juvenile hormone treatment on larval begging (withinsubjects contrast: $F_{1,29}=6.18, P=0.019$ ), confirming that there was indeed a general increase in larval begging as a function of increasing juvenile hormone concentration (Fig. 1). The quadratic component of the function describing the effect of juvenile hormone treatment on larval begging was nonsignificant (within-subjects contrast: $\left.F_{1,29}=0.20, P=0.66\right)$.

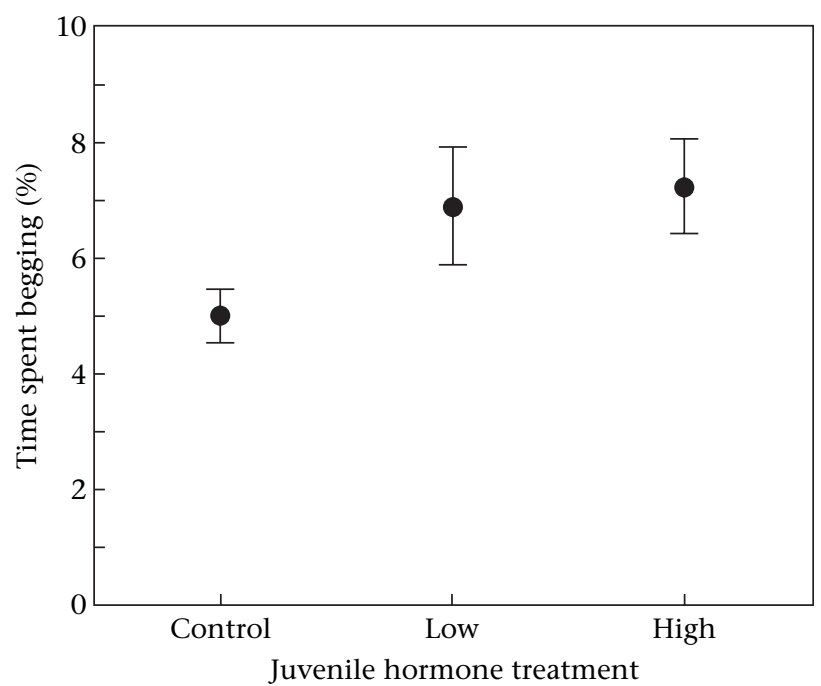

Figure 1. Mean \pm SE time spent begging of $N$. vespilloides larvae $6 \mathrm{~h}$ after treatment with the juvenile hormone analogue methoprene. Control refers to the solvent control treatment (i.e. $0.5 \mu \mathrm{l}$ of $95 \%$ ethanol), low refers to the low juvenile hormone treatment (i.e. $25 \mu \mathrm{g}$ of methoprene in $0.5 \mu \mathrm{l}$ of $95 \%$ ethanol), and high refers to the high juvenile hormone treatment (i.e. $50 \mu \mathrm{g}$ of methoprene in $0.5 \mu \mathrm{l}$ of $95 \%$ ethanol).

\section{Larval Growth}

Juvenile hormone treatment had a highly significant effect on larval growth among larvae that begged for resources from parents (repeated measures GLM: withinsubjects effects: Greenhouse-Geisser correction: $F_{3.0,57.9}=$ 14.55, $P<0.001)$. In contrast to our initial expectation, juvenile hormone treatment had a negative effect on larval growth (Fig. 2a).

Juvenile hormone treatment also had a highly significant negative effect on larval growth among larvae that foraged by self-feeding only following the removal of parents (repeated measures GLM: within-subjects effects: Greenhouse-Geisser correction: $F_{2.7,51.9}=9.84, P<0.001$; Fig. 2b). Thus, juvenile hormone treatment had a negative effect on larval growth regardless of whether the larvae foraged by begging or self-feeding.

An analysis of the combined data for larvae that foraged by begging in the presence of parents and by self-feeding in the absence of parents confirmed that juvenile hormone treatment had indeed a highly significant negative effect on larval growth (repeated measures GLM: withinsubjects effects: Greenhouse-Geisser correction: $F_{3.0,114.7}=$ 22.75, $P<0.001$; Fig. 2). There was no significant effect of the interaction between the presence and absence of parents and juvenile hormone treatment $\left(F_{3.0,114.7}=0.55\right.$, $P=0.65$; Fig. 2). Thus, there was no evidence that juvenile hormone treatment had a differential effect on growth among larvae that foraged by begging and larvae that foraged by self-feeding only. Trend analysis revealed a significant linear effect of juvenile hormone treatment on larval growth (within-subjects contrast: $F_{1,38}=14.83$, $P<0.001)$, confirming that there was indeed a general decrease in larval growth as a function of increasing juvenile hormone concentration (Fig. 2). The quadratic 


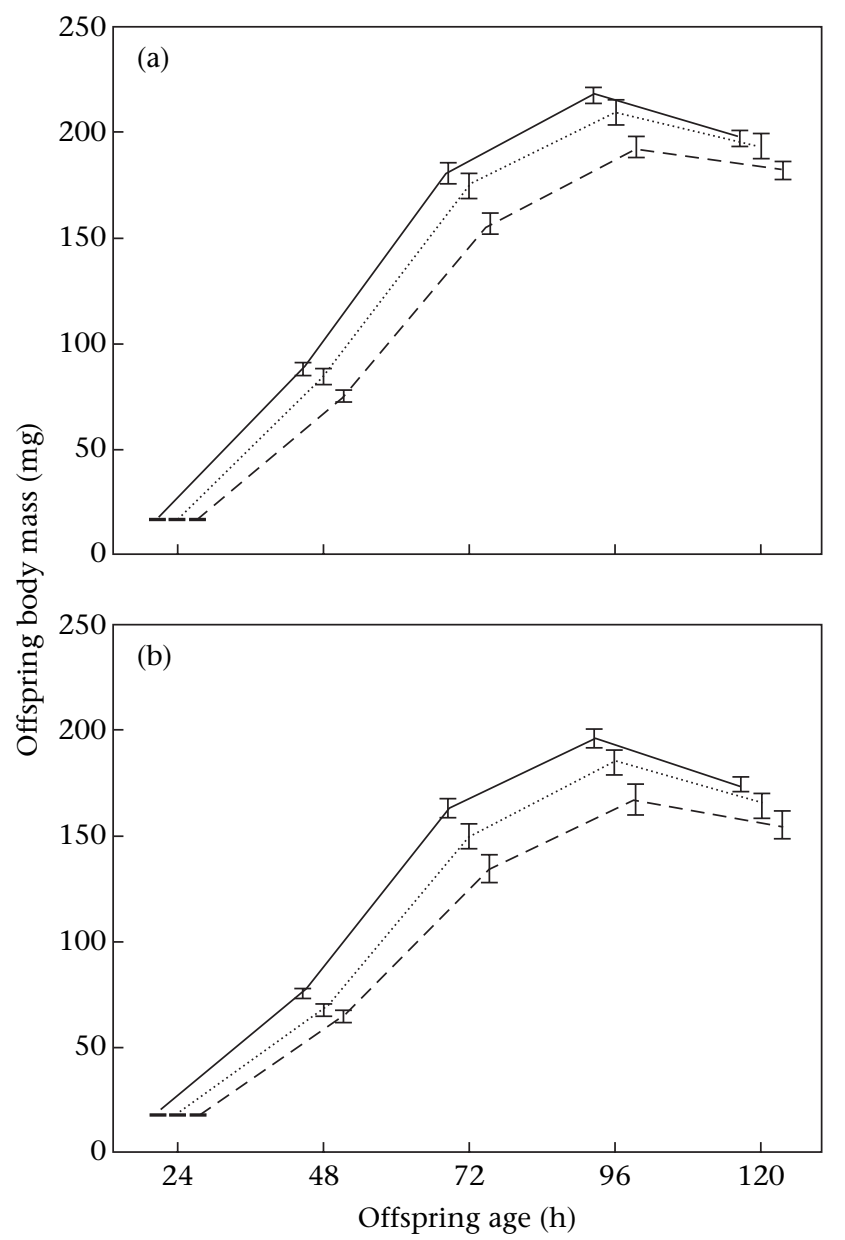

Figure 2. Mean \pm SE body mass of $N$. vespilloides larvae from age of treatment with the juvenile hormone analogue methoprene ( $24 \mathrm{~h})$, and at consecutive $24 \mathrm{~h}$ intervals until reaching maximum body mass $(96 \mathrm{~h})$. Solid lines represent the solvent control treatment (i.e. $0.5 \mu \mathrm{l}$ of $95 \%$ ethanol), dotted lines represent the low juvenile hormone treatment (i.e. $25 \mu \mathrm{g}$ of methoprene in $0.5 \mu \mathrm{l}$ of $95 \%$ ethanol), and dashed lines represent the high juvenile hormone treatment (i.e. $50 \mu \mathrm{g}$ of methoprene in $0.5 \mu \mathrm{l}$ of $95 \%$ ethanol). (a) Body mass of larvae that could forage by begging from the parents. (b) Body mass of larvae that foraged by self-feeding only.

component of the function describing the effect of juvenile hormone treatment on larval begging was nonsignificant (within-subjects contrast: $F_{1,38}=0.31, P=0.58$ ), suggesting that juvenile hormone treatment had a graded effect on larval growth.

\section{DISCUSSION}

Here, we have shown that experimentally elevated levels of juvenile hormone stimulate begging behaviour in N. vespilloides larvae, thus providing the first evidence that hormones may be involved in the modulation of larval begging in insects. Thus, although juvenile hormone is structurally and often functionally different from testosterone and corticosterone (Nijhout 1994), our study suggests that juvenile hormone could play a similar role in modulating larval begging in insect larvae as testosterone and corticosterone in nestling birds (Schwabl 1996; Kitaysky et al. 2001; Goodship \& Buchanan 2006). Further studies are needed to verify the suggestion that juvenile hormone plays a role in the modulation of larval begging in insects. In particular, there is a need for information on the dynamics of juvenile hormone titers in begging $N$. vespilloides larvae. Such information is currently lacking owing to the technical difficulties of measuring juvenile hormone titers.

Birds and insects are very distant taxa with distinct hormone systems (Nijhout 1994). Thus, our study raises the interesting possibility that hormonal modulation of offspring begging may have evolved independently in the two taxa due to similar selection pressures. At present, the evolution of hormonal modulation of begging is poorly understood in any species. In general, selection is expected to favour hormonal modulation of social behaviour whenever the expression of a given behaviour varies with age, competitive rank, sex, season or environmental condition (Adkins-Regan 2005). This condition is met for both birds and insects because begging levels in both taxa change as a function of offspring age (e.g. Smiseth et al. 2003; Leonard \& Horn 2006) and differ between competitively superior (i.e. early-hatched) and inferior (i.e. latehatched) offspring (e.g. Cotton et al. 1999; Smiseth \& Moore, in press).

We found that juvenile hormone treatment reduced larval growth, contradicting our initial expectation that juvenile hormone would enhance growth. Our initial expectation was based on studies on birds showing that maternally derived testosterone enhances nestling growth (Schwabl 1996; Pilz et al. 2004). Interestingly, some studies on birds report that maternally derived testosterone reduces growth (e.g. Sockman \& Schwabl 2000), suggesting that hormones that stimulate begging can have negative as well as positive effects on growth. One potential mechanism by which hormones could reduce growth is to increase the costs of inefficient begging (Pilz et al. 2004). We can exclude this mechanism as an explanation for our findings because juvenile hormone had similar effects on growth of larvae that foraged by self-feeding and by begging. Further research is needed to understand why juvenile hormone reduces growth of $N$. vespilloides. Three potential explanations for why juvenile hormone may reduce growth are that juvenile hormone (1) increases metabolic activity (Sláma \& Hodková 1975), (2) suppresses the growth of imaginal tissues (Emlen \& Allen 2003), or (3) suppresses immune function (Rantala et al. 2003) which, in turn, may influence growth due to trade-offs between immune function and growth (Rantala \& Roff 2005).

The relationships between a given hormone, offspring begging, and growth are complex because begging affects growth by increasing access to resources, while begging in turn reflects the offspring's nutritional state (Kilner \& Johnstone 1997). In many birds, testosterone and corticosterone are thought to promote growth indirectly by stimulating begging, thereby increasing the nestlings' access to resources (Schwabl 1996; Kitaysky et al. 2001; Goodship \& Buchanan 2006). This scenario cannot account for our finding that juvenile hormone in $N$. vespilloides decreased growth regardless of whether larvae foraged by begging or self-feeding. Instead, this finding suggests that juvenile 
hormone reduces growth directly instead of or in addition to stimulating begging. Reduced growth may in turn stimulate begging because begging reflects the offspring's nutritional condition (Kilner \& Johnstone 1997; Smiseth \& Moore 2004).

The modulation of larval begging might represent a novel effect of juvenile hormone, adding to the numerous aspects of insect physiology, development, life history, and behaviour that are affected by this remarkably versatile hormone (Nijhout 1994; Flatt et al. 2005). Previous studies showed that juvenile hormone is positively correlated with parental care towards begging larvae in burying beetles (Panaitof et al. 2004; Scott \& Panaitof 2004; Trumbo \& Robinson 2004). Thus, juvenile hormone may have a positive effect on both sides of parentoffspring communication. Interestingly, offspring begging and parental care are positively genetically correlated in $N$. vespilloides (Lock et al. 2004). Because hormones such as juvenile hormone might be major determinants of genetic correlations (e.g. Flatt \& Kawecki 2004; Flatt et al. 2005), the genetic correlation between offspring begging and parental care could be due to pleiotropic effects of genes involved in juvenile hormone signalling.

In conclusion, we have shown that juvenile hormone affects larval begging and growth in insects, suggesting that juvenile hormone could have a similar effect as maternally derived or endogenous testosterone and corticosterone in birds. However, our results suggest that the specific mechanisms by which hormones affect begging and growth differ between insects and birds. To address this issue, further studies are needed on the mechanisms by which hormones affect begging and growth in different taxa. Furthermore, there is a need for studies that directly address the evolution of hormonal effects on begging and growth. Recently, the evolution of hormone regulation has been studied directly in Gryllus crickets using a quantitative genetics approach (Zera 2006). Thus, insects may provide particularly tractable models for studying the evolution of hormonal effects on offspring begging and growth.

\section{Acknowledgments}

We thank the Mersey Valley Countryside Warden Service and Alan Barton for giving us permission to collect beetles on their property at Sunbank Wood, Manchester. We thank Kate Buchanan, Diego Gil, Ed Harris, Becky Kilner, Richard Preziosi, Markus Rantala, Steve Trumbo and an anonymous referee for valuable comments on the manuscript. The study was financially supported by a NERC Postdoctoral Fellowship to P.T.S. and by a Swiss National Science Foundation Postdoctoral Fellowship to T.F.

\section{References}

Adkins-Regan, E. 2005. Hormones and Animal Social Behavior. Princeton, New Jersey: Princeton University Press.

Cotton, P. A., Wright, J. \& Kacelnik, A. 1999. Chick begging strategies in relation to brood hierarchies and hatching asynchrony. American Naturalist, 153, 412-420.
Eggert, A.-K. \& Müller, J. K. 1997. Biparental care and social evolution in burying beetles: lessons from the larder. In: The Evolution of Social Behavior in Insects and Arachnids (Ed. by J. E. Choe \& B. J. Crespi), pp. 216-236. Cambridge: Cambridge University Press.

Eising, C. M. \& Groothius, T. G. G. 2003. Yolk androgens and begging behaviour in black-headed gull chicks: an experimental field study. Animal Behaviour, 66, 1027-1034.

Emlen, D. J. \& Allen, C. E. 2003. Genotype to phenotype: physiological control of trait size and scaling in insects. Integrative and Comparative Biology, 43, 617-634.

von Ende, C. N. 2001. Repeated-measures analysis. In: Design and Analysis of Ecological Experiments (Ed. by S. M. Scheiner \& J. Gurevitch), pp. 134-157. New York: Oxford University Press.

Flatt, T. \& Kawecki, T. J. 2004. Pleiotropic effects of methoprenetolerant (Met), a gene involved in juvenile hormone metabolis$\mathrm{m}$, on life history traits in Drosophila melanogaster. Genetica, 122, 141-160.

Flatt, T., Tu, M. P. \& Tatar, M. 2005. Hormonal pleiotropy and the juvenile hormone regulation of Drosophila development and life history. Bioessays, 27, 999-1010.

Godfray, H. C. J. 1991. Signalling of need by offspring to their parents. Nature, 352, 328-330.

Godfray, H. C. J. 1995. Signaling of need between parents and young: parent-offspring conflict and sibling rivalry. American Naturalist, 146, 1-24.

Goodship, N. M. \& Buchanan, K. L. 2006. Nestling testosterone is associated with begging behaviour and fledging success in the pied flycatcher, Ficedula hypoleuca. Proceedings of the Royal Society of London, Series B, 273, 71-76.

Howell, D. C. 2002. Statistical Methods for Psychology. 5th edn. Belmont, California: Duxbury Press.

Kaptein, N., Billen, J. \& Gobin, B. 2005. Larval begging for food enhances reproductive options in the ponerine ant Gnamptogenys striatula. Animal Behaviour, 69, 293-299.

Kilner, R. \& Johnstone, R. A. 1997. Begging the question: are offspring solicitation behaviours signals of need? Trends in Ecology \& Evolution, 12, 11-15.

Kitaysky, A. S., Wingfield, J. C. \& Piatt, J. F. 2001. Corticosterone facilitates begging and affects resource allocation in black-legged kittiwakes. Behavioral Ecology, 12, 619-625.

Kölliker, M., Chuckalovcak, J. P., Haynes, K. F. \& Brodie, E. D., III 2006. Maternal food provisioning in relation to condition-dependent offspring odours in burrower bugs (Sehinus cinctus). Proceedings of the Royal Society of London, Series B, 273, 1523-1528.

Leonard, M. L. \& Horn, A. G. 2006. Age-related changes in signalling of need by nestling tree swallows (Tachycineta bicolor). Ethology, 112, 1020-1026.

Lock, J. E., Smiseth, P. T. \& Moore, A. J. 2004. Selection, inheritance and the evolution of parent-offspring interactions. American Naturalist, 164, 13-24.

Martin, P. \& Bateson, P. 1986. Measuring Behaviour: an Introductory Guide. Cambridge: Cambridge University Press.

Nijhout, H. F. 1994. Insect Hormones. Princeton, New Jersey: Princeton University Press.

Panaitof, S. C., Scott, M. P. \& Borst, D. W. 2004. Plasticity in juvenile hormone in male burying beetles during breeding: physiological consequences of the loss of a mate. Journal of Insect Physiology, 50, 715-724.

Pilz, K., Quiroga, M., Schwabl, H. \& Adkins-Regan, E. 2004. European starling chicks benefit from high yolk testosterone levels during a drought year. Hormones and Behavior, 46, 179-192.

Rantala, M. J. \& Roff, D. A. 2005. An analysis of trade-offs in immune function, body size and development time in the Mediterranean field cricket, Gryllus bimaculatus. Functional Ecology, $19,323-330$. 
Rantala, M. J., Vainikka, A. \& Kortet, R. 2003. The role of juvenile hormone in immune function and pheromone production tradeoffs: a test of the immunocompetence handicap principle. Proceedings of the Royal Society of London, Series B, 270, 2257-2261.

Rauter, C. M. \& Moore, A. J. 1999. Do honest signalling models of offspring solicitation apply to insects? Proceedings of the Royal Society of London, Series B, 266, 1691-1696.

Rauter, C. M. \& Moore, A. J. 2002. Quantitative genetics of growth and development time in the burying beetle Nicrophorus pustulatus in the presence and absence of post-hatching parental care. Evolution, 56, 96-110.

Schal, C., Holbrook, G. I., Bachmann, J. A. S. \& Sevala, V. I. 1997. Reproductive biology of the German cockroach, Blatella germanica: juvenile hormone as a pleiotropic master regulator. Archives of Insect Biochemistry and Physiology, 35, 405-426.

Schwabl, H. 1996. Maternal testosterone in the avian egg enhances postnatal growth. Comparative Biochemistry and Physiology, Part A, 114, 271-276.

Scott, M. P. 1998. The ecology and behavior of burying beetles. Annual Review of Entomology, 43, 595-618.

Scott, M. P. \& Panaitof, S. C. 2004. Social stimuli affect juvenile hormone during breeding in biparental burying beetles (Silphidae: Nicrophorus). Hormones and Behavior, 45, 159-167.

Sláma, K. \& Hodková, M. 1975. Insect hormones and bioanalogues: their effect on respiratory metabolism in Dermestes vulpinus L. (Coleoptera). Biological Bulletin, 148, 320-332.

Smiseth, P. T. \& Moore, A. J. 2002. Does resource availability affect offspring begging and parental provisioning in a partially begging species? Animal Behaviour, 63, 577-585.
Smiseth, P. T. \& Moore, A. J. 2004. Signalling of hunger when offspring forage by both begging and self-feeding. Animal Behaviour, 67, 1083-1088.

Smiseth, P. T. \& Moore, A. J. In press. Signalling of need by senior and junior larvae in asynchronous broods of the burying beetle Nicrophorus vespilloides. Animal Behaviour.

Smiseth, P. T., Darwell, C. T. \& Moore, A. J. 2003. Partial begging: an empirical model for the early evolution of offspring signalling. Proceedings of the Royal Society of London, Series B, 270, 1773-1777.

Smiseth, P. T., Dawson, C., Varley, E. \& Moore, A. J. 2005. How do caring parents respond to mate loss? Differential response by males and females. Animal Behaviour, 69, 551-559.

Sockman, K. W. \& Schwabl, H. 2000. Yolk androgens reduce offspring survival. Proceedings of the Royal Society of London, Series $B, 267,1451-1456$.

Trumbo, S. T. \& Robinson, G. E. 2004. Nutrition, hormones and life history in burying beetles. Journal of Insect Physiology, 50, 383-391.

Williams, C. M. 1956. The juvenile hormone of insects. Nature, 178, 212-213.

Wilson, T. G. 2004. The molecular site of action of juvenile hormone and juvenile hormone insecticides during metamorphosis: how these compounds kill insects. Journal of Insect Physiology, 50, 111-121.

Zera, A. J. 2006. Evolutionary genetics of juvenile hormone and ecdysteroid regulation in Gryllus: a case study in the microevolution of endocrine regulation. Comparative Biochemistry and Physiology, Part A, 144, 365-379. 